\title{
MIMO Ricean Channel Capacity: An Asymptotic Analysis
}

\author{
Guillaume Lebrun, Michael Faulkner, Mansoor Shafi, and Peter J. Smith
}

\begin{abstract}
This paper presents asymptotic bounds and limits for the mean channel capacity of MIMO systems under Ricean channel conditions. It is shown that the mean capacity per dimension decreases as the $K$ factor increases in value and approaches a value equal to that of the underlying scattering channel when the number of antennas are large and the specular matrix has unit rank. The accuracy of the bounds is verified by simulations. In addition, a variety of results for the MIMO Ricean channel are brought together to give an overview of the current knowledge in this area. We also show that the variance of the capacity for a Ricean Channel approaches that of the the scattering channel for large numbers of antennas.
\end{abstract}

Index Terms-MIMO, Ricean channel, capacity.

\section{INTRODUCTION}

$\mathbf{S}_{\mathrm{n} i \mathrm{~s}}$ INCE the work of Foschini [1] and Telatar [2], there has been intense research activity in the area of MIMO systems. Most of this research effort has been focussed on the flat-fading Rayleigh channel, which corresponds to a wireless propagation environment where the number of scatterers is large. It is now well understood that the capacity of the MIMO Rayleigh channel increases linearly with the number of antennas for a fixed ratio of transmit to receive antenna numbers. Therefore, it is convenient to define a normalized capacity, the capacity of the channel divided by the minimum of the number of antennas at the receiver and the number of antennas at the transmitter.

It has been reported recently that the standardized Rayleigh channel capacity tends to a Gaussian random variable as the number of transmit and receive antennas tends to infinity [3]. Further, the capacity distribution is close to Gaussian even for small antenna numbers. A good summary of asymptotic results for the independent Rayleigh case is given in [4]. In the Rayleigh case, asymptotic results provide useful results and insights even for moderate numbers of antennas.

In this paper we consider the ergodic capacity for the more general case of a Ricean channel. In the Ricean case, the flat-fading channel is composed of a Line Of Sight (LOS) component and a Rayleigh component. The choice of the Ricean $K$-factor varies the Ricean channel from a Rayleigh channel $(K=-\infty \mathrm{dB})$ to a pure LOS channel $(K=+\infty$

Manuscript received November 19, 2003; revised April 18, 2005; accepted April 26, 2005. The associate editor coordinating the review of this letter and approving it for publication was Y.-C. Liang.

Guillaume Lebrun and Michael Faulkner are with the Australian Telecommunication CRC Centre for Telecom. and Micro-electronics, Victoria University, Melbourne, Australia (e-mail: \{lebrun, mf $\} @$ sci.vu.edu.au)

Mansoor Shafi is with Telecom New Zealand Ltd, Wellington, New Zealand (e-mail: \{mansoor.shafi\}@ telecom.co.nz)

Peter J. Smith is with the Dept. of Electrical and Computer Eng., University of Canterbury, Christchurch, New Zealand (e-mail: \{p.smith\}@elec.canterbury.ac.nz)

Digital Object Identifier 10.1109/TWC.2006.03647.
dB). It has been shown [5] that the MIMO capacity decreases with increasing power of the LOS component (the $K$-factor). The main contributions of this paper are:

- we show that the ergodic normalized capacity of the Ricean MIMO channel approaches the corresponding normalized capacity of the underlying scattering channel when the antenna numbers are large.

- We show that the capacity variance of the Ricean channel approaches the corresponding variance of the underlying scattering channel when the antenna numbers are large.

- We develop upper and lower bounds for the ergodic capacity of the Ricean Channel and discuss their characteristics for different $K$ values. We confirm the accuracy of the bounds via simulation.

In addition, we discuss the eigenvalue analysis of the channel gain matrix and its constituent components. This provides useful insights into the asymptotic behavior of the capacity of the MIMO Ricean channel. Note that these results are derived for the commonly considered case where the specular matrix is of unit rank. It has been shown [5] that for special array geometries, giving a full rank specular matrix, the pure Ricean capacity can behave like the Rayleigh case. Hence it is likely that results for the rank $>1$ situation will be case specific.

The outline of the paper is as follows. Section II describes the system model. The capacity of LOS and Rayleigh channels is discussed in Section III; Section III-D discusses correlated channels. Section IV gives the main capacity bounds and results that are verified by simulation in Section V.

\section{SYSTEM MODEL}

Consider a single-user MIMO system. Transmission is over a flat-fading Ricean channel with $t$ antennas at the transmitter and $r$ antennas at the receiver. If $\boldsymbol{x}$ is a vector of input symbols $\left(\boldsymbol{x} \in \mathbb{C}^{t}\right), \boldsymbol{H}$ the channel matrix $\left(\boldsymbol{H} \in \mathbb{C}^{r \times t}\right)$, and $\boldsymbol{n}$ a vector of additive white Gaussian noise (AWGN) on the receiving antennas $\left(\boldsymbol{n} \in \mathbb{C}^{r}\right)$, the vector of received symbols can be expressed as

$$
\boldsymbol{y}=\boldsymbol{H} \boldsymbol{x}+\boldsymbol{n} .
$$

In Ricean fading the elements of $\boldsymbol{H}$ are non-zero mean complex Gaussians. Hence we can express $\boldsymbol{H}$ in matrix notation as [6]

$$
\boldsymbol{H}=a \boldsymbol{H}^{s p}+b \boldsymbol{H}^{s c}
$$

where the specular and scattered components of $\boldsymbol{H}$ are denoted by superscripts, $a>0, b>0$ and $a^{2}+b^{2}=1$. The entries of $\boldsymbol{H}^{s c}=\left(h_{i, j}\right)$ are independent and identically distributed (iid) complex Gaussian random variables with zero mean and unit magnitude variance. A common model for $\boldsymbol{H}^{s p}$ 
is $\boldsymbol{H}^{s p}=\boldsymbol{v}_{r} \times \boldsymbol{v}_{t}^{*}$ [6] where $\boldsymbol{v}_{r}$ and $\boldsymbol{v}_{t}$ have modulus one elements and are the specular array responses at the receiver and the transmitter respectively. The superscript ${ }^{*}$ denotes the transpose conjugate. Here, we assume $\boldsymbol{H}^{s p}$ is an $r \times t$ matrix of unit entries. Note this assumption is without loss of generality, since the statistics of $\boldsymbol{H} \boldsymbol{H}^{*}$, required for the capacity, depend on $\boldsymbol{H}^{s p}$ only through the eigenvalues of $\boldsymbol{H}^{s p}\left(\boldsymbol{H}^{s p}\right)^{*}$. Also, the choice of unit entries gives exactly the same eigenvalues for $\boldsymbol{H}^{s p}\left(\boldsymbol{H}^{s p}\right)^{*}$ as the physical model given by $\boldsymbol{v}_{r} \times \boldsymbol{v}_{t}^{*}$. The Ricean $K$-factor is defined as $10 \log _{10}\left(a^{2} / b^{2}\right) \mathrm{dB}$.

Assuming equal power uncorrelated sources the capacity is

$$
C=\log _{2}\left(\operatorname{det}\left(\boldsymbol{I}_{r}+\frac{\rho}{t} \boldsymbol{H} \boldsymbol{H}^{*}\right)\right),
$$

where $\boldsymbol{I}_{r}$ is the identity matrix of dimension $r$ and $\rho$ is the SNR on each receiving antenna. The ergodic capacity is commonly defined as the expected value of $C$ in (3). The normalized capacity is defined as the ergodic capacity divided by $m$ where $m \triangleq \min (t, r)$. Recently, there has been good progress in providing bounds on the Ricean capacity in (3) [8], [9] and some exact results derived from multivariate statistics are also available [10]. In this paper we investigate the complementary issue of asymptotic results.

\section{CAPACITY OF RAYLEIGH AND LOS ChanNELS}

In this Section we summarize results on the extreme cases of a pure LOS channel and a pure Rayleigh channel as well as the effects of correlation in a MIMO channel. Some new results are also given to complete the summary.

\section{A. Pure LOS Channel : $K=+\infty$}

In general, a MIMO LOS channel has a capacity of

$$
C(K=+\infty, t, r, \rho)=\log _{2}(1+\rho r) .
$$

Since the channel is not random, the capacity is fixed and the ergodic capacity and the capacity are equal. It should be noted that the capacity does not depend on the number of transmit antennas, and only increases logarithmically with the number of receive antennas. In the special case $t=r=1$, the channel reduces to a Single Input Single Output (SISO) Additive White Gaussian Noise (AWGN) channel.

\section{B. Pure Rayleigh Channel : $K=-\infty, r=1$ or $t=1$}

For the Rayleigh channel, $\left\|\boldsymbol{H}_{i, j}\right\|^{2}$ is a $\chi_{2}^{2}$ variate (chisquared variate with two degrees of freedom) but normalized so that $E\left(\left\|\boldsymbol{H}_{i, j}\right\|^{2}\right)=1$, where $E($.$) denotes the expectation$ and $\|$.$\| denotes the absolute value. For one transmit antenna,$ the channel capacity is [1]

$$
C(K=-\infty, t=1, r, \rho)=\log _{2}\left(1+\rho \chi_{2 r}^{2}\right),
$$

and using one receive antenna the channel capacity is [1]

$$
C(K=-\infty, t, r=1, \rho)=\log _{2}\left(1+(\rho / t) \chi_{2 t}^{2}\right) .
$$

Notice that

$$
\begin{aligned}
E\left(1+(\rho / t) \chi_{2 t}^{2}\right) & =(1+\rho) \\
E\left(1+\rho \chi_{2 r}^{2}\right) & =(1+\rho r),
\end{aligned}
$$

and $\log _{2}(\cdot)$ is a concave function, that is $\forall z>0 E\left(\log _{2}(z)\right) \leq$ $\log _{2}(E(z))$. Therefore

$$
E(C(K=-\infty, t, r=1, \rho)) \leq C(K=+\infty, t, r=1, \rho)
$$

and

$$
E(C(K=-\infty, t=1, r, \rho)) \leq C(K=+\infty, t=1, r, \rho),
$$

Hence, for a Single Input Multiple Output (SIMO) or Multiple Input Single Output (MISO) channel, the ergodic capacity is higher in a LOS case than in a Rayleigh case (see Fig. 3).

\section{C. $K=-\infty, r \rightarrow \infty, t \rightarrow \infty$ and $t / r=\alpha$}

When $\boldsymbol{H}$ is Rayleigh $(K=-\infty)$ and the number of antennas is large, the normalized capacity can be approximated by a Gaussian random variable [3]. Suppose $r \rightarrow \infty, t \rightarrow \infty$ with $t / r=\alpha$, then the mean is given by [4]

$$
\begin{aligned}
E(C / m)= & \left(\log _{2}\left(w_{+} \rho\right)+\ldots\right. \\
& \left.(1-\alpha) \log _{2}\left(1-w_{-}\right)-\frac{w_{-} \alpha}{\ln 2}\right) \max (1, \beta) .
\end{aligned}
$$

where $m \triangleq \min (t, r), \beta \triangleq 1 / \alpha$,

$$
w_{ \pm} \triangleq\left(w \pm \sqrt{w^{2}-4 / \alpha}\right) / 2
$$

and

$$
w \triangleq 1+\frac{1}{\alpha}+\frac{1}{\rho}
$$

The variance of $\mathrm{C}$ is also given in [4] as,

$$
\sigma_{C}^{2}=-\log _{2} e \log _{2}\left\|1-\frac{q^{2} p^{2}}{\beta}\right\|
$$

with

$$
\begin{aligned}
& q \triangleq \frac{\sqrt{\rho}}{2}\left(\beta-1-1 / \rho+\sqrt{(\beta-1-\rho)^{2}}+4 \beta / \rho\right) \\
& p \triangleq \frac{\sqrt{\rho}}{2}\left(1-\beta-1 / \rho+\sqrt{(1-\beta-\rho)^{2}}+4 / \rho\right) .
\end{aligned}
$$

\section{Correlation and Correlation Models}

It is well-known that MIMO capacity in Ricean channels is reduced by increasing the $\mathrm{K}$-factor [5], [6], since the increasing importance of the LOS path creates a larger "common" component in the channel gains and diversity is reduced. In this sense, the effects of LOS paths in Ricean channels and correlation in Rayleigh channels are equivalent. Results for the Ricean case are fairly limited, whereas a large body of knowledge has been compiled in recent years on the capacity of correlated channels. Hence, for reasons of completeness, we summarize this work below. Note that correlation does not always reduce capacity [11] but for many commonly used correlation models, such as the Kronecker model [35], it does have this negative effect.

The choice of a correlation model is critical in the analysis of the effect of correlation on the capacity of MIMO channels. Though the most widespread correlation model is arguably the one ring model [13], other models include the exponential correlation matrix [14], [15] and the virtual representation channel matrix [16]-[18]. Closed form functions for the correlation of MIMO channels have also been derived [19], [20]. A fading model that enables the characterization of signal correlation is given in [21], and a MIMO macrocellular broadband channel 
model is given in [22]. Models in this area are being developed very rapidly. A detailed description of the correlated MIMO channel can be found in [12].

\section{E. Correlation: Field Measurements}

Several measurement campaigns have been conducted to determine the correlation properties of real-life MIMO channels. The impact and extent of correlation on indoor MIMO channels is discussed in [23]-[26]. In [27] it is shown that the MIMO capacity increases as the signal correlation decreases but the SNR has a greater impact than correlation on the MIMO capacity as shown in [28]. Outdoor correlation measurements are discussed in [29], [30]. MIMO standard models were derived from field measurements [31].

\section{F. Correlation: Generic results}

Early results on the effect of correlation focused on its effect on specific systems, such as adaptive arrays [32] or maximum ratio combining systems [33]. The effects of fading correlation have been studied for the Rayleigh channel through simulation [34] and analysis [13], [35]-[37]. Further results investigated the impact of correlation on the time variation of capacity [38]. Mutual coupling between radiating elements is one source of correlation. Its effect on capacity is studied in [39].

\section{G. Correlation: Asymptotic Results and Bounds on Capacity}

An asymptotic approach to describing MIMO capacity under correlated fading is described in [40], [41]. A closed form upper bound is derived in [42] when long term CSI is available at the transmitter. An upper bound on mean capacity is given in [43]-[45].

\section{H. Correlation: Ricean Channel}

Simulation results for the capacity of the Ricean channel are presented in [46]. A geometric approach to interpret the capacity is described in [5] and simulations in [3] indicate that the capacity can be approximated by a Gaussian random variable. Analytical results on the capacity are now emerging for finite numbers of antennas [7] or in the special case of the low power regime [47].

\section{CAPACITY BOUNDS FOR THE RICEAN CHANNEL}

For large numbers of antennas, it has been suggested in the literature that the capacity of the Ricean channel tends to the capacity of its Rayleigh component [48] and that the capacity can be upper bounded by the sum of the capacities of the Rayleigh and LOS component matrices [49].

In this Section we look at the limiting case where $r \rightarrow \infty$, $t \rightarrow \infty$ and $t / r=\alpha$. We derive lower and upper bounds for Ricean channel capacity. Since the Rayleigh capacity grows linearly with $m$ and the LOS capacity only grows logarithmically, it is intuitively obvious that the normalized Ricean ergodic capacity will approach that of the underlying Rayleigh channel especially when the number of antennas $(t, r)$ grows large. Also the Ricean ergodic capacity should be greater than that of the underlying Rayleigh channel. Neither of these results appear to be available and so we prove them in this Section. In order to understand the asymptotic capacity behaviour for the Ricean channel it is instructive to study the eigenvalues of $\boldsymbol{H}$.

To begin, note that

$$
\log _{2}\left|\boldsymbol{I}_{r}+\frac{\rho}{t} \boldsymbol{H} \boldsymbol{H}^{*}\right|=\log _{2}\left|\boldsymbol{I}_{r}+\frac{b^{2} \rho}{t} \boldsymbol{H}^{s c}\left(\boldsymbol{H}^{s c}\right)^{*}+\frac{\rho}{t} \boldsymbol{F}\right|,
$$

with $a, b$ defined by (2) and $\boldsymbol{F}$ is the $r \times r$ hermitian matrix,

$$
\boldsymbol{F}=a b\left(\boldsymbol{H}^{s c}\left(\boldsymbol{H}^{s p}\right)^{*}+\boldsymbol{H}^{s p}\left(\boldsymbol{H}^{s c}\right)^{*}\right)+a^{2} \boldsymbol{H}^{s p}\left(\boldsymbol{H}^{s p}\right)^{*} .
$$

\section{A. Study of the Eigenvalues of $\boldsymbol{F}$}

We note that $\boldsymbol{F}$ consists of two parts: a cross product term due to the specular and scattering channel gains and a specular term. The former is itself a sum of two terms. The eigenvalues of $\boldsymbol{F}$ provide useful insights to understanding the MIMO Ricean capacity. We assume throughout this analysis that $a \neq 0$.

1) Singular Value Decompositions: The matrices $\boldsymbol{H}^{s p}\left(\boldsymbol{H}^{s p}\right)^{*}$ and $\boldsymbol{H}^{s c}\left(\boldsymbol{H}^{s p}\right)^{*}$ can be written,

$$
\boldsymbol{H}^{s p}\left(\boldsymbol{H}^{s p}\right)^{*}=t \times(\mathbf{1})_{r, r} .
$$

and

$$
\boldsymbol{H}^{s c}\left(\boldsymbol{H}^{s p}\right)^{*}=\left(\sum_{k=1}^{t} h_{i, k}\right)_{i=1 . . r, j=1 . . r} .
$$

Both are rank one matrices and have the following singular value decompositions,

$$
\begin{aligned}
\boldsymbol{H}^{s p}\left(\boldsymbol{H}^{s p}\right)^{*} & =\left(\overrightarrow{v_{1}}\right) r t\left({\overrightarrow{v_{1}}}^{*}\right) \\
\boldsymbol{H}^{s c}\left(\boldsymbol{H}^{s p}\right)^{*} & =\left(\overrightarrow{u_{1}}\right) \sigma\left({\overrightarrow{v_{1}}}^{*}\right) .
\end{aligned}
$$

The singular values are $r t$ and $\sigma$ and the singular vectors are $\overrightarrow{v_{1}}$ and $\overrightarrow{u_{1}}$. These are defined below,

$$
\overrightarrow{v_{1}}=\frac{1}{\sqrt{r}}(\mathbf{1})_{r, 1}
$$

The singular vector $\overrightarrow{u_{1}}$ is given by $\overrightarrow{u_{1}}=\overrightarrow{x_{1}} /\left\|x_{1}\right\|$, where

$$
\begin{gathered}
\overrightarrow{x_{1}}=\left(\sum_{k=1}^{t} h_{1, k}, \sum_{k=1}^{t} h_{2, k}, \ldots, \sum_{k=1}^{t} h_{r, k}\right)^{T}, \\
\sigma=\sqrt{r \times \sum_{i=1}^{r}\left\|\sum_{k=1}^{t} h_{i, k}\right\|^{2} .}
\end{gathered}
$$

2) Eigenvalues of $\boldsymbol{F}$ : Using the singular value decompositions above, we can write $\boldsymbol{F}$ in (16) as $\boldsymbol{F}=a^{2} r t\left(\vec{v}_{1}\right)\left(\vec{v}_{1}^{*}\right)+$ $a b \sigma\left(\left(\overrightarrow{v_{1}}\right)\left(\vec{u}_{1}^{*}\right)+\left(\overrightarrow{u_{1}}\right)\left(\vec{v}_{1}^{*}\right)\right)$. Hence $\operatorname{rank}(\boldsymbol{F}) \leq 2$ since $\boldsymbol{F}$ is the sum of two rank 1 matrices, $a^{2} r t\left(\overrightarrow{v_{1}}\right)\left({\overrightarrow{v_{1}}}^{*}\right)+a b \sigma\left(\overrightarrow{v_{1}}\right)\left(\vec{u}_{1}{ }^{*}\right)$ and $a b \sigma\left(\overrightarrow{u_{1}}\right)\left(\vec{v}_{1}^{*}\right)$. By construction, it follows that any eigenvector, $\vec{k}$, of $\boldsymbol{F}$, associated with the non-zero eigenvalue $\kappa$ satisfies the following,

$$
\left\{\begin{array}{l}
\exists \beta_{1}, \beta_{2} \text { such that } \vec{k}=\beta_{1} \overrightarrow{v_{1}}+\beta_{2} \overrightarrow{u_{1}} \\
\boldsymbol{F} \vec{k}=\kappa \vec{k},
\end{array}\right.
$$




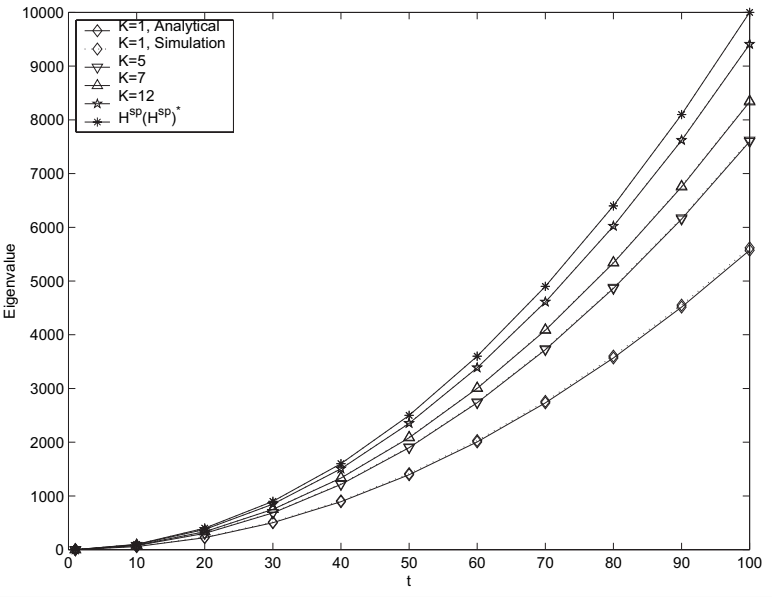

Fig. 1. Positive eigenvalue of $\boldsymbol{F}$ versus antenna numbers for various $K$ values

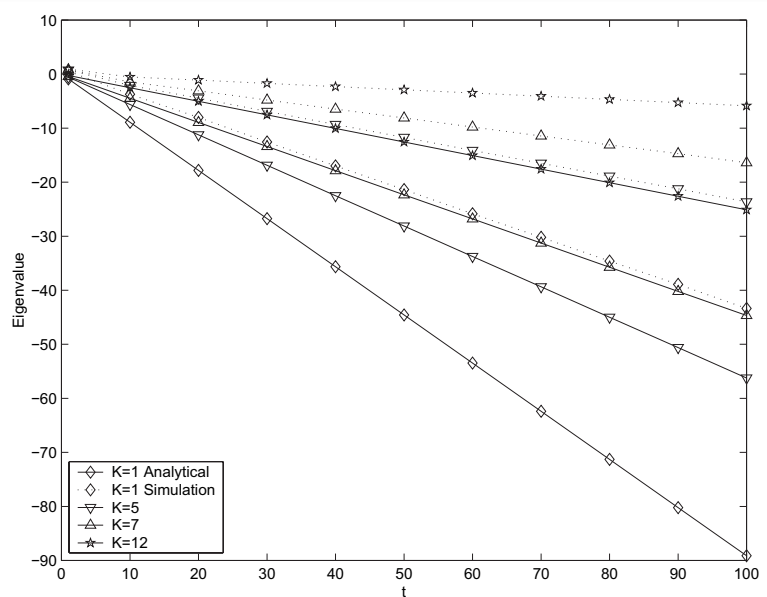

Fig. 2. Negative eigenvalue of $\boldsymbol{F}$ versus antenna numbers for various $K$ values

Subsituting for $\boldsymbol{F}$ and $\vec{k}$ in $\boldsymbol{F} \vec{k}=\kappa \vec{k}$ gives an equation involving linear combinations of $\overrightarrow{v_{1}}$ and $\overrightarrow{u_{1}}$. Equating the coefficients of these vectors gives:

$\left\{\begin{array}{l}\beta_{1} a b \sigma\left(\overrightarrow{u_{1}}{ }^{*} \overrightarrow{v_{1}}\right)+\beta_{2} a b \sigma+\beta_{1} a^{2} r t+\beta_{2} a^{2} r t\left({\overrightarrow{v_{1}}}^{*} \overrightarrow{u_{1}}\right)=\kappa \beta_{1} \\ \beta_{1} a b \sigma+\beta_{2} a b \sigma\left({\overrightarrow{v_{1}}}^{*} \overrightarrow{u_{1}}\right)=\kappa \beta_{2}\end{array}\right.$

Defining $o={\overrightarrow{v_{1}}}^{*} \overrightarrow{u_{1}}$, and solving (25) for $\kappa$ gives,

$$
\kappa=\frac{\frac{a^{2} r t+a b \sigma\left(o+o^{*}\right)}{2} \pm \ldots}{\frac{\sqrt{\left(a^{2} r t+a b \sigma\left(o+o^{*}\right)\right)^{2}+4(a b \sigma)^{2}\left(1-o o^{*}\right)}}{2} .}
$$

which defines the 2 possibly non-zero eigenvalues of $\boldsymbol{F}$.

3) Asymptotic eigenvalues of $\boldsymbol{F}$ : Equation (23) indicates that $\sigma \geq 0$ and $E\left(\sigma^{2}\right)=r^{2} \times t$. Furthermore, $\|o\| \leq 1$, so for $r, t \rightarrow \infty,\left(a^{2} r t+a b \sigma\left(o+o^{*}\right)\right)^{2} \gg 4(a b \sigma)^{2}\left(1-o o^{*}\right)$ and $a^{2} r t \gg\left\|a b \sigma\left(o+o^{*}\right)\right\|$ with probability 1 . Hence, one solution of (26) is positive and the other negative. Since, all other eigenvalues are zero we have the ordered eigenvalues denoted by $\lambda_{r}(\boldsymbol{F})<0=\lambda_{r-1}(\boldsymbol{F})=\ldots=\lambda_{2}(\boldsymbol{F})<\lambda_{1}(\boldsymbol{F})$. Taking the positive square root in (26) gives $\lambda_{1}(\boldsymbol{F}) \sim a^{2} r t+$ $a b \sigma\left(o+o^{*}\right)$ and in the limit

$$
\lambda_{1}(\boldsymbol{F}) / r t \rightarrow a^{2} .
$$

From equation (26), $\boldsymbol{F}$ is a matrix of maximum rank two, with one negative and one positive eigenvalue. The positive eigenvalue, denoted $\lambda_{1}(\boldsymbol{F})$, behaves as in (27) and hence grows quadratically with the number of antennas (since $a^{2} r t=$ $\left.a^{2} \alpha r^{2}\right)$. Despite this, we expect $\boldsymbol{F}$ to have a negligible effect in (15) for large numbers of antennas, since $\boldsymbol{F}$ only has 2 eigenvalues whereas the scattering term has $m$. The two eigenvalues of $\boldsymbol{F}$ are shown in Fig. 1 and Fig. 2.

Now the following further observations can be made:

- The positive eigenvalue is several orders of magnitude larger than the magnitude of the negative eigenvalue. Its growth with $t, r(t=r)$ is quadratic.

- The disparity between the sizes of the positive and negative eigenvalues increases even further when the $K$ value is such that the channel is effectively a LOS channel.

From results not reported here we also remark that the eigenvalues of the sum of the cross product terms in (16) is a matched pair of positive and negative terms. The effect of the term $\boldsymbol{H}^{s p}\left(\boldsymbol{H}^{s p}\right)^{*}$ in (16) is to cause the large increase in the positive eigenvalue seen in Fig. 1.

Whilst the positive eigenvalue may seem quite large, its contribution to capacity is relatively small due to the logarithmic operation.

\section{B. Capacity Lower bound}

We now derive the capacity lower bound. Since $\boldsymbol{H} \boldsymbol{H}^{*}$ is a non-central complex Wishart matrix we can use Bartlett's decomposition described in [50] for the real non-central case. A simple extension to the complex case gives

$$
\boldsymbol{H} \boldsymbol{H}^{*}=b^{2} \boldsymbol{L}^{*} \boldsymbol{L}
$$

where $\boldsymbol{L}$ is upper triangular with diagonal elements denoted $L_{1}, L_{2}, \ldots L_{r}$ which are independent of all other elements. We assume that $r \leq t$ but the proof can easily be adapted to $r>t$ also. The distribution of $L_{1}^{2}$ is non-central chi-squared, $L_{1}^{2} \sim \chi_{2 t}^{2}(\delta)$ with $\delta=\left(a^{2} / b^{2}\right) \operatorname{trace}\left(\boldsymbol{H}^{s p}\left(\boldsymbol{H}^{s p}\right)^{*}\right)$. For $j>$ 1 the distributions are central chi-squared, $L_{j}^{2} \sim \chi_{2 t-2 j+2}^{2}$. Hence, we have

$$
D=\left|\boldsymbol{I}_{r}+\frac{\rho}{t} \boldsymbol{H} \boldsymbol{H}^{*}\right|=\left|\left[\boldsymbol{I}_{r} \sqrt{\frac{b^{2} \rho}{t}} \boldsymbol{L}^{*}\right]\left[\begin{array}{c}
\boldsymbol{I}_{r} \\
\sqrt{\frac{b^{2} \rho}{t}} \boldsymbol{L}
\end{array}\right]\right|
$$

Using the Cauchy-Binet theorem gives

$$
D=\sum_{\gamma}\left|\boldsymbol{A}_{\gamma}\right|\left|\boldsymbol{A}_{\gamma}\right|^{*}=\sum_{\gamma}\left|\boldsymbol{A}_{\gamma}\right|^{2},
$$

where $\boldsymbol{A}_{\gamma}$ is an $r \times r$ submatrix of $\left[\boldsymbol{I}_{r} \sqrt{\frac{b^{2} \rho}{t}} \boldsymbol{L}^{*}\right]$ and $\gamma$ is a subset of $r$ columns from $(1,2, \ldots, 2 r)$.

Now we split the summation into two parts, over $\gamma_{1}$ where the determinants $\left|\boldsymbol{A}_{\gamma_{1}}\right|$ do not involve $L_{1}$ and over $\gamma_{2}$ where the determinants $\left|\boldsymbol{A}_{\gamma_{2}}\right|$ do involve $L_{1}$. Hence

$$
D=\sum_{\gamma_{1}}\left|\boldsymbol{A}_{\gamma_{1}}\right|^{2}+\sum_{\gamma_{2}}\left|\boldsymbol{A}_{\gamma_{2}}\right|^{2}
$$

The only choice of columns which gives determinants involving $L_{1}$ are those where column $r+1$ is selected and column 1 is omitted. Hence the $\boldsymbol{A}_{\gamma_{2}}$ matrices are of the form 


$$
\boldsymbol{A}_{\gamma_{2}}=\left[\begin{array}{ccccccc}
0 & \ldots & 0 & \sqrt{\frac{b^{2} \rho}{t}} L_{1} & 0 & \ldots & 0 \\
& & 0 & & \\
& D_{\gamma_{21}} & \vdots & & D_{\gamma_{22}} \\
& & 0 & &
\end{array}\right]
$$

Hence $\left|\boldsymbol{A}_{\gamma_{2}}\right|^{2}=\frac{b^{2} \rho}{t} L_{1}^{2}\left|D_{\gamma_{2}}\right|^{2}$ where $D_{\gamma_{2}}=\left[D_{\gamma_{21}} D_{\gamma_{22}}\right]$ and $D=\sum_{\gamma_{1}}\left|\boldsymbol{A}_{\gamma_{1}}\right|^{2}+\frac{b^{2} \rho}{t} L_{1}^{2} \sum_{\gamma_{2}}\left|D_{\gamma_{2}}\right|^{2}=X+L_{1}^{2} Y$. Exactly the same analysis holds for the Rayleigh case, except $L_{1}^{2} \sim$ $\chi_{2 t}^{2}$.

To summarize,

$$
\begin{gathered}
D^{\text {Ricean }}=X+\chi_{2 t}^{2}(\delta) Y \\
D^{\text {Rayleigh }}=X+\chi_{2 t}^{2} Y
\end{gathered}
$$

where $X, Y$ are positive random variables with $X, Y$ independent of the $\chi^{2}$ variables. Hence

$$
\begin{aligned}
& E(C(\boldsymbol{H}))=E\left(\log _{2}(X)\right)+E\left(\log _{2}\left(1+\chi_{2 t}^{2}(\delta) Y / X\right)\right) \\
& E\left(C\left(b \boldsymbol{H}^{s c}\right)\right)=E\left(\log _{2}(X)\right)+E\left(\log _{2}\left(1+\chi_{2 t}^{2} Y / X\right)\right) .
\end{aligned}
$$

Now $\chi_{2 t}^{2}(\delta)$ is stochastically greater than $\chi_{2 t}^{2}$. Hence $E\left(f\left(\chi_{2 t}^{2}(\delta)\right)\right) \geq E\left(f\left(\chi_{2 t}^{2}\right)\right)$ for any increasing function $f$ and $E(C(\boldsymbol{H})) \geq E\left(C\left(b \boldsymbol{H}^{s c}\right)\right)$ as required. We are now able to write a lower bound for the capacity , $\forall r, t, \rho, K$,

$$
E\left(\frac{C(K, t, r, \rho)}{\min (t, r)}\right) \geq E\left(\frac{C\left(K=-\infty, t, r, b^{2} \rho\right)}{\min (t, r)}\right) .
$$

\section{Capacity Upper bound}

We shall now derive the capacity upper bound. Defining

$$
\boldsymbol{A}=\boldsymbol{I}_{r}+\frac{b^{2} \rho}{t} \boldsymbol{H}^{s c}\left(\boldsymbol{H}^{s c}\right)^{*},
$$

we have the normalized capacity as

$$
\frac{C}{t}=\frac{1}{t} \log _{2}(|\boldsymbol{A}+\tilde{\boldsymbol{F}}|)=\frac{1}{t} \log _{2}\left(\prod_{i=1}^{r} \lambda_{i}(\boldsymbol{A}+\tilde{\boldsymbol{F}})\right),
$$

where $\tilde{\boldsymbol{F}}=\frac{\rho}{t} \boldsymbol{F}$ and $\lambda_{i}(\boldsymbol{A}+\tilde{\boldsymbol{F}})$ are the eigenvalues of the hermitian positive definite matrix $\boldsymbol{A}+\tilde{\boldsymbol{F}}$, ordered so that $0 \leq$ $\lambda_{r}(\boldsymbol{A}+\tilde{\boldsymbol{F}}) \leq \ldots \leq \lambda_{1}(\boldsymbol{A}+\tilde{\boldsymbol{F}})$. Combining Weyl's theorem [51] and results from Section IV-A leads to

$$
\begin{aligned}
& \lambda_{r}(\boldsymbol{A}+\tilde{\boldsymbol{F}}) \leq \lambda_{r-1}(\boldsymbol{A})+\lambda_{2}(\tilde{\boldsymbol{F}})=\lambda_{r-1}(\boldsymbol{A}) \\
& \lambda_{r-1}(\boldsymbol{A}+\tilde{\boldsymbol{F}}) \leq \lambda_{r-2}(\boldsymbol{A})+\lambda_{2}(\tilde{\boldsymbol{F}})=\lambda_{r-2}(\boldsymbol{A}) \\
& \vdots \\
& \lambda_{2}(\boldsymbol{A}+\tilde{\boldsymbol{F}}) \leq \lambda_{1}(\boldsymbol{A})+\lambda_{2}(\tilde{\boldsymbol{F}})=\lambda_{1}(\boldsymbol{A}) \\
& \lambda_{1}(\boldsymbol{A}+\tilde{\boldsymbol{F}}) \leq \lambda_{1}(\boldsymbol{A})+\lambda_{1}(\tilde{\boldsymbol{F}})
\end{aligned}
$$

Therefore,

$$
\begin{aligned}
\frac{C}{t} & \leq \frac{1}{t} \log _{2}\left(\lambda_{r-1}(\boldsymbol{A}) \ldots \lambda_{1}(\boldsymbol{A})\left(\lambda_{1}(\boldsymbol{A})+\lambda_{1}(\tilde{\boldsymbol{F}})\right)\right. \\
& =\frac{1}{t} \log _{2}\left(\prod_{i=1}^{r} \lambda_{i}(\boldsymbol{A})\right)+\frac{1}{t} \log _{2}\left(\frac{\lambda_{1}(\boldsymbol{A})+\lambda_{1}(\tilde{\boldsymbol{F}})}{\lambda_{r}(\boldsymbol{A})}\right) .
\end{aligned}
$$

Now write

$$
\Delta=\frac{1}{t} \log _{2}\left(\frac{\lambda_{1}(\boldsymbol{A})+\lambda_{1}(\tilde{\boldsymbol{F}})}{\lambda_{r}(\boldsymbol{A})}\right),
$$

and, since $\lambda_{j}(\boldsymbol{A}) \geq 1$ for any $\mathrm{j}$,

$$
\Delta \leq \frac{1}{t} \log _{2}\left(\lambda_{1}(\boldsymbol{A})+\frac{\rho}{t} \lambda_{1}(\boldsymbol{F})\right)
$$

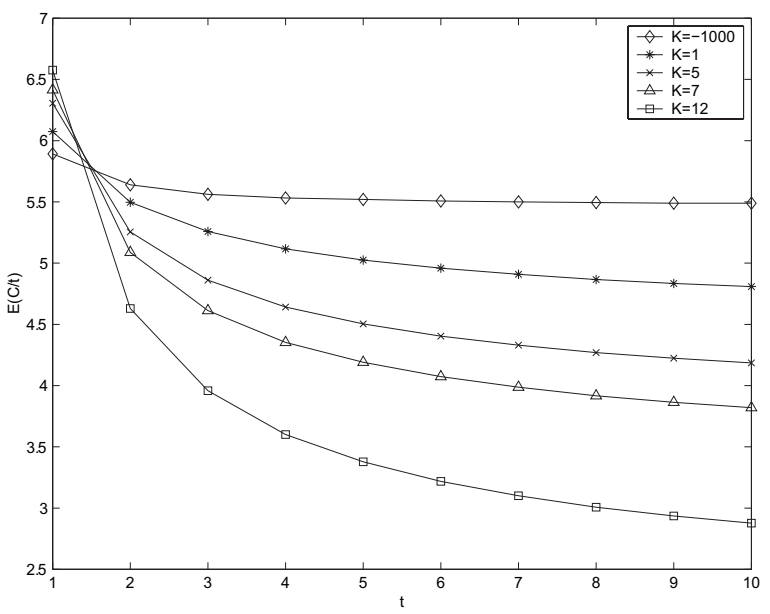

Fig. 3. Normalized mean capacity per antenna with $t / r=1$, Ricean fading and $\mathrm{SNR}=20 \mathrm{~dB}$

It is known that the eigenvalues of $\boldsymbol{A}$ are bounded as $r, t \rightarrow \infty$ [52]. Therefore, $\exists M$ such that $\lambda_{1}(\boldsymbol{A}) \leq M$ and when $r, t \rightarrow$ $\infty$

$$
\Delta \leq \frac{1}{t} \log _{2}\left(M+\rho r\left(\lambda_{1}(\boldsymbol{F}) /(r t)\right)\right) \rightarrow 0,
$$

since $\lambda_{1}(\boldsymbol{F}) /(r t) \rightarrow a^{2}$. This concludes the demonstration. From [52] we know that

$$
\lambda_{1}(\boldsymbol{A}) \rightarrow 1+b^{2} \rho(1+\sqrt{\min (t, r) / \max (t, r)})^{2},
$$

as $t, r \rightarrow \infty$ with $t / r=\alpha$. This provides the smallest value for $M$ that can be used and gives the bound that we use in the simulations. Note that our approach assumes that we can interchange the order of integration and the limit in $\lim _{r, t \rightarrow \infty} E(\Delta)$. This is valid by the dominated convergence theorem since $\operatorname{abs}(\Delta)$ has a finite mean.

Therefore we can write the capacity upper bound as $t, r \rightarrow$ $\infty$

$$
E\left(\frac{C(K, t, r, \rho)}{\min (t, r)}\right) \leq E\left(\frac{C\left(K=-\infty, t, r, b^{2} \rho\right)}{\min (t, r)}\right)+\Delta
$$

where $\Delta \rightarrow 0$ as $t, r \rightarrow \infty$.

Hence, for Ricean channels that are not pure LOS $(K \neq$ $+\infty$ ), the normalized ergodic capacity tends to the normalized ergodic capacity of the scattering component. Hence,

$$
E\left(\frac{C(K, t, r, \rho)}{\min (t, r)}\right) \rightarrow E\left(\frac{C\left(K=-\infty, t, r, b^{2} \rho\right)}{\min (t, r)}\right) .
$$

\section{RESUlts AND Discussion}

\section{A. Mean and Variance of Capacity for the Ricean Channel}

Fig. 3 plots the average normalized capacity with $\alpha=1$ for an increasing number of antennas and different $K$-factors. As indicated in our analysis, for $t=1$, the mean capacity of Ricean channels is higher than the mean capacity of Rayleigh channels. This trend is inverted for $t, r>1$. This result is in contrast with the outage capacity of the Ricean channel which remains higher than the corresponding capacity of the Rayleigh channel for small numbers of antennas (i.e. up to four antennas) when the targeted outage probability is very small (i.e. 0.01) [46]. Such a result is not surprising since the 


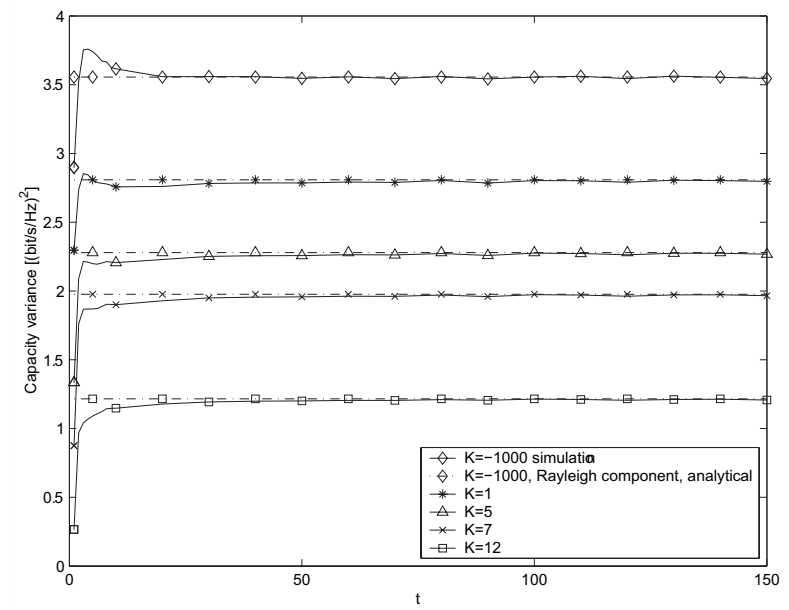

Fig. 4. Capacity variance with $t / r=1$, Ricean fading and $\mathrm{SNR}=20 \mathrm{~dB}$

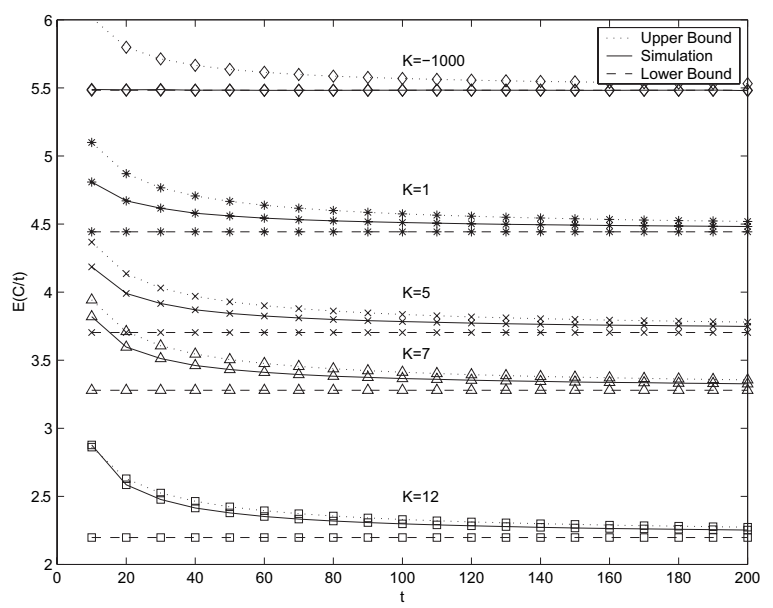

Fig. 5. Ergodic capacity per antenna with $t, r \rightarrow \infty$, Ricean fading and $\mathrm{SNR}=20 \mathrm{~dB}$

capacity of the Ricean channel is a random variable with a smaller variance than the capacity of the Rayleigh channel [3].

For $K=-1000$, the capacity converges rapidly to a limit as $t \rightarrow \infty$, as indicated in [4]. For other values of $K$, the capacity decreases with the number of antennas over this range. As soon as $t>1$, the capacity of the Ricean channel is a decreasing function of $K$. The results shown in Fig. 3 are for $t=r$.

Fig. 4 plots the variance of the total capacity for different $K$ values versus the numbers of transmit and receive antennas when $S N R=20 \mathrm{~dB}$ and $\alpha=1$. For the purposes of comparison the corresponding analytical results by [4] is also shown for the Rayleigh Channel. From these results it is clear that under Ricean channel conditions the limiting variance is equal to that of the underlying Rayleigh channel.

\section{B. Asymptotic Mean and Variance of the Capacity for the Ricean Channel}

Fig. 5 shows the behaviour of the normalized capacity of a Ricean channel as the number of antennas grows large. For all values of $K$, the normalized capacity of the Ricean channel tends to the normalized capacity of its scattering component

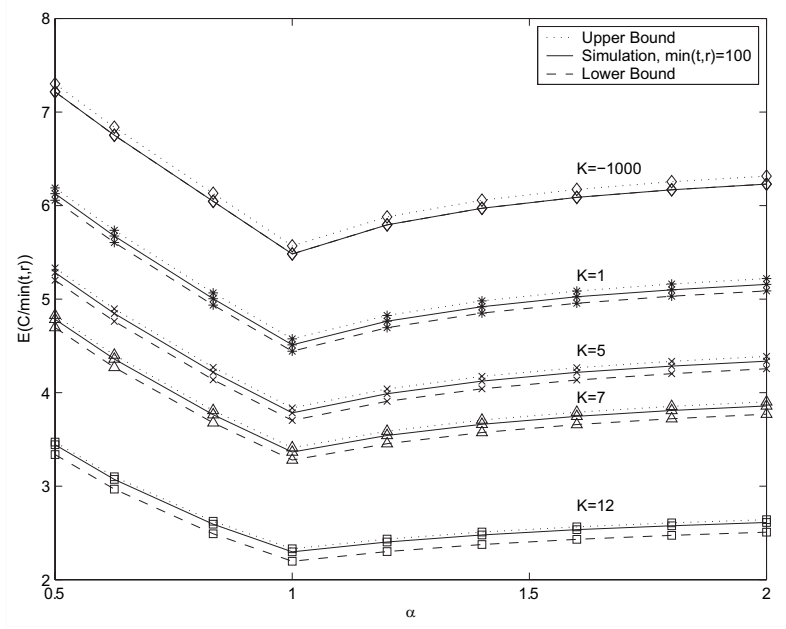

Fig. 6. Ergodic capacity per antenna versus $\alpha$, Ricean fading and $\mathrm{SNR}=20 \mathrm{~dB}$

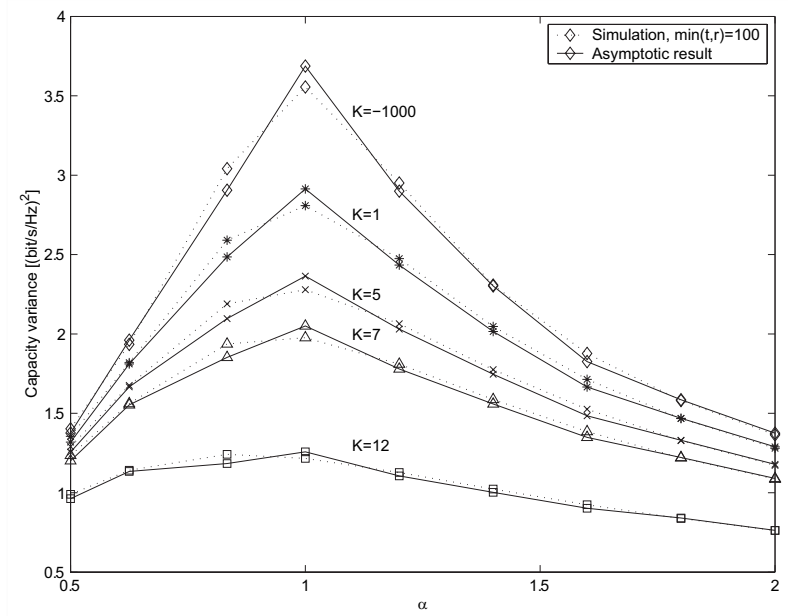

Fig. 7. Asymptotic capacity variance, Ricean fading and $\mathrm{SNR}=20 \mathrm{~dB}$

(the lower bound on the capacity). This lower bound is tighter when $K$ is smaller, and for $K=-1000$ it is impossible to discern the simulation from the lower bound.

The upper bound converges slowly to the lower bound and is tight for large values of $K$. An explanation for the slowness of convergence can be found in (42) where it is shown that $\Delta$ tends to zero like $\log (r) / t$, which itself converges very slowly. Although the upper bound is only strictly valid for a large number of antennas (see the assumptions in (43)), in the simulations it is still correct for values of $t$ as low as 20, and for $K \leq 12$.

The capacity bounds for values of $\alpha$ other than 1 are shown in Fig. 6. The tightness of both bounds remains unaffected for any value of $\alpha$.

The results shown in Fig. 4 are further reinforced by Fig. 7. This shows the behavior of the variance of the capacity of a Ricean channel as the number of antennas grows large. For all values of $K$, the variance clearly tends to the corresponding variance of its scattering component, the variance is maximum for $\alpha=1$ and the maximum decreases as the channel approaches LOS condition. We note from results not reported here that this asymptotic behavior of the capacity variance is only achieved for very large number of antennas, i.e. $t=r=$ 100 or more. 


\section{Capacity Bounds}

Fig. 6 shows the behaviour of the normalized capacity, for varying $\alpha$, in the asymptotic case of a large number of antennas $(\min (t, r)=100)$. As in Fig. 5, the lower bound is tight for small $K$, whereas the upper bound is tight for large $K$. Note that the tightness of the bounds appears uniform across all $\alpha$ values, indicating that the tightness depends on the ratio $t / r$, and not on their actual values.

The results demonstrate that the upper and lower bounds provide a fast and reliable way to bound the mean capacity of a Ricean channel for large $m$, without extensive simulations. Furthermore, depending on the $K$-factor, it is straightforward to deduce which of the bounds is the tightest.

\section{CONCLUSIONS}

The capacity of the Rayleigh and LOS channels have been studied extensively and are well known, both for a small number of antennas and in the asymptotic case of a large number of antennas. For a Ricean channel, the capacity is more difficult to derive.

For a large number of antennas, the normalized mean capacity of a Ricean channel tends to the normalized mean capacity of its Rayleigh component. Precisely, the capacity of the Ricean channel is lower bounded by the capacity of its Rayleigh component and upper bounded by a quantity that tends to the capacity of its Rayleigh component when the number of antennas grows large.

The lower bound is valid for any number of antennas, and depending on the choice of a constant $M$, the upper bound can be valid for any number of antennas, or only for a large number of antennas (in which case the upper bound is tighter when the number of antennas grows large). The lower bound is tighter when the $K$-factor is smaller, whereas the upper bound is tighter with increasing $K$. The two bounds allow us to estimate the capacity of a Ricean channel without extensive simulations.

A very similar behavior is observed by the capacity variance which also tends to the corresponding variance of the scattering component when the number of antennas grows large.

The asymptotic results of the Ricean Channel are useful to observe the speed of convergence towards asymptotic behavior when moderate antenna numbers are employed.

\section{REFERENCES}

[1] G. J. Foschini and M. J. Gans, "On limits of wireless communication in a fading environment when using multiple antennas," Wireless Personal Communications, vol. 6, no. 3, pp. 311-335, Mar. 1998

[2] I. E. Telatar, "Capacity of multi-antenna Gaussian channels," European Transactions on Telecommunications, vol. 10, no. 6, pp. 585-595, 1999.

[3] P. J. Smith and M. Shafi, "On a Gaussian approximation to the capacity of wireless MIMO systems," in Proc. IEEE International Conference on Communications (ICC2002), pp. 406-410, New York, Apr. 28-May 2, 2002.

[4] E. Biglieri and G. Tarico, "Large-System Analysis of Multiple-Antenna System capacities," Journal of Communications and Networks., vol. 5, no. 2, pp. 96-103, June. 2003.

[5] P. F. Driessen and G. J. Foschini, "On the capacity formula for multipleinput-multiple-output wireless channels: a geometric approach," IEEE Trans. Commun., vol. 47, no. 2, pp. 173-176, Feb. 1999.

[6] F. R. Farrokhi, G. J. Foschini, A. Lozano and R. A. Valenzuela, "Link-optimal space-time processing with multiple transmit and receive antennas," IEEE Commun. Lett., vol. 5, no. 3, pp. 85-87, Mar. 2001.
[7] P. J. Smith and L. M. Garth, "Exact capacity distribution for Dual MIMO systems in Ricean fading," in press IEEE Commun. Lett. .

[8] S. K. Jayaweera and H. V. Poor, "On the capacity of multi-antenna systems in the presence of Rician fading," in Proc. IEEE Vehicular Technology Conference (VTC 2002 Fall), vol. 4, pp. 1963-1967, 24-28 Sept. 2002

[9] S. K. Jayaweera and H. V. Poor, "MIMO capacity results for Rician fading channels," in Proc. IEEE Global Telecommunications Conference (GLOBECOM 03), vol 4, pp. 1806-1810, 1-5 Dec. 2003.

[10] M. Kang and M. Alouini, "Capacity of MIMO Rician channels," in Proc 40th Annual Conference on Communication, Control and Computing, Monticello, Il, Oct. 2002.

[11] C. Oestges and A. J. Paulraj, "Beneficial impact of channel correlations on MIMO capacity," Electronics Letters, vol. 40, no. 10, pp. 606-608, May 2004.

[12] D. Asztely, "On antenna arrays in mobile communication systems: fast fading and GSM base station receiver algorithms," Technical Report IR S3-SB-9611, Royal Institute of Technology, stockholm, Sweden, Mar, 19996.

[13] D. Chizhik, F. Rashid-Farrokhi, J. Ling, and A. Lozano, "Effect of antenna separation on the capacity of BLAST in correlated channels," IEEE Commun. Lett., vol. 4, no. 11, pp. 337-339, Nov. 2000.

[14] S.L. Loyka, "Channel capacity of MIMO architecture using the exponential correlation matrix," IEEE Commun. Lett., vol. 5, Issue: 9, pp. 369-371, Sept. 2001.

[15] S. Loyka and G. Tsoulos, "Estimating MIMO system performance using the correlation matrix approach," IEEE Commun. Lett., vol. 6, Issue: 1, pp. 19-21, Jan. 2002.

[16] V. V. Veeravalli, A. Sayeed, and Yingbin Liang, "Asymptotic capacity of correlated MIMO Rayleigh fading channels via virtual representation," in Proc. IEEE International Symposium on Information Theory, pp. 247, June 29-July 42003.

[17] V. Raghavan and A. M. Sayeed, "MIMO capacity scaling and saturation in correlated environments," in Proc. IEEE International Conference on Communications (ICC '03), vol. 5, pp. 3006-3010, 2003.

[18] Z. Hong, K. Liu, R. W. Heath Jr., and A. M. Sayeed, "Spatial multiplexing in correlated fading via the virtual channel representation," IEEE J. Select. Areas Commun., vol. 21, no. 5, pp. 856-866, June 2003.

[19] G. J. Byers and F. Takawira, "The influence of spatial and temporal correlation on the capacity of MIMO channels," in Proc. IEEE Wireless Communications and Networking Conference, vol. 1, pp. 359-364, 16-20 Mar. 2003.

[20] A. Abdi and M. Kaveh, "Space-time correlation modeling of multielement antenna systems in mobile fading channels," in Proc. 2001 IEEE International Conference on Acoustics, Speech, and Signal Processing (ICASSP '01), vol. 4, pp. 2505-2508, 7-11 May 2001.

[21] A. Abdi and M. Kaveh, "A space-time correlation model for multielement antenna systems in mobile fading channels," IEEE J. Select. Areas Commun., vol. 20, Issue: 3, pp. 550-560, Apr. 2002.

[22] C. Oestges, V. Erceg, and A. J. Paulraj, "A physical scattering model for MIMO macrocellular broadband wireless channels," IEEE J. Select. Areas Commun., vol. 21, Issue: 5, pp. 721-729, June 2003.

[23] M. Stoytchev and H. Safar, "Statistics of the MIMO radio channel in indoor environments," in Proc. IEEE Vehicular Technology Conference (VTC 2001 Fall), vol. 3, pp. 1804-1808, 7-11 Oct. 2001.

[24] P. Kyritsi and D. C. Cox, "Correlation properties of MIMO radio channels for indoor scenarios," Conference Record of the Thirty-Fifth Asilomar Conference on Signals, Systems and Computers, vol. 2, pp. 994-998, 4-7 Nov. 2001.

[25] D. P. McNamara, M. A. Beach and P. N. Fletcher, "Spatial correlation in indoor MIMO channels," The 13th IEEE International Symposium on Personal, Indoor and Mobile Radio Communications (PIMRIC 2002), vol. 1, pp. 290-294, 15-18 Sept. 2002.

[26] J. P. Kermoal, L. Schumacher, P. E. Mogensen, and K. I. Pedersen, "Experimental investigation of correlation properties of MIMO radio channels for indoor picocell scenarios," in Proc. IEEE Vehicular Technology Conference (VTC 2000 Fall), vol. 1, pp. 14-21, 24-28 Sept. 2000.

[27] P. Kyritsi, D. C. Cox, R. A. Valenzuela, and P. W. Wolniansky, "Correlation analysis based on MIMO channel measurements in an indoor environment," IEEE J. Select. Areas Commun., vol. 21, Issue: 5, pp. 713-720, June 2003.

[28] D. P. McNamara, M. A. Beach, P.N. Fletcher and P. Karlsson, "Capacity variation of indoor multiple-input multiple-output channels," Electronics Letters, vol. 36, Issue: 24, pp. 2037-2038, 23 Nov 2000.

[29] P. Soma, D. S. Baum, V. Erceg, R. Krishnamoorthy, and A. J. Paulraj, "Analysis and modeling of multiple-input multiple-output (MIMO) radio channel based on outdoor measurements conducted at $2.5 \mathrm{GHz}$ for 
fixed BWA applications," in Proc. IEEE International Conference on Communications (ICC 2002), vol. 1, pp. 272-276, 28 April-2 May 2002.

[30] D. Chizhik, J. Ling, P. W. Wolniansky, R. A. Valenzuela, N. Costa, and K. Huber, "Multiple-input-multiple-output measurements and modeling in Manhattan," IEEE J. Select. Areas Commun., vol. 21, Issue: 3, pp. 321-331, April 2003.

[31] D. P. Palomar, J. R. Fonollosa, and M. A. Lagunas, "Capacity results of spatially correlated frequency-selective MIMO channels in UMTS," in Proc. IEEE Vehicular Technology Conference (VTC 2001 Fall), vol. 2, pp. 553-557, 7-11 Oct. 2001.

[32] J. Salz and J. H. Winters, "Effect of fading correlation on adaptive arrays in digital mobile radio," IEEE Trans. Veh. Technol., vol. 43, Issue: 4, pp. 1049-1057, Nov. 1994.

[33] M. Kang and M. Alouini, "Impact of correlation on the capacity of MIMO channels," in Proc. IEEE International Conference on Communications (ICC '03), vol. 4, pp. 2623-2627, 2003.

[34] Chen-Nee Chuah, J.M. Kahn and D. Tse, "Capacity of multi-antenna array systems in indoor wireless environment," Proc. IEEE Global Telecommunications Conference (GLOBECOM 98), vol 4, pp. 18941899, 8-12 Nov. 1998.

[35] D.-S. Shiu, G. J. Foschini, M. J. Gans, and J. M. Kahn, "Fading correlation and its effect on the capacity of multielement antenna systems," IEEE Trans. Commun., vol. 48, no. 3, pp. 502-513, Mar. 2000.

[36] Chen-Nee Chuah, D.N.C. Tse, J.M. Kahn and R.A. Valenzuela, "Capacity scaling in MIMO wireless systems under correlated fading," IEEE Trans. Inform. Theory, vol. 48, no. 3, pp. 637-650, Mar. 2002.

[37] C. Martin and B. Ottersten, "Analytic approximations of eigenvalue moments and mean channel capacity for MIMO channels," in Proc. IEEE International Conference on Acoustics, Speech, and Signal Processing (ICASSP '02), vol. 3, pp. III2389-III2392, 13-17 May 2002.

[38] A. Giorgetti, M. Chiani, M. Shafi, and P. J. Smith, "Level crossing rates and MIMO capacity fades: Impacts of spatial/temporal channel correlation," in Proc. IEEE International Conference on Communications (ICC '03), vol. 5, pp. 3046-3050, 2003.

[39] P. N. Fletcher, M. Dean, and A. R. Nix, "Mutual coupling in multielement array antennas and its influence on MIMO channel capacity," Electronics Letters, vol. 39, Issue: 4, pp. 342-344, 20 Feb 2003.

[40] X. Mestre and J.R. Fonollosa, "Effect of fading correlation on the asymptotic open-loop and closed-loop capacity of MIMO systems," in Proc. IEEE Information Theory Workshop, pp. 349-352, 31 Mar.-4 Apr. 2003. 01216765

[41] X. Mestre, J. R. Fonollosa, and A. Pages-Zamora, "Capacity of MIMO channels: asymptotic evaluation under correlated fading," IEEE J. Select. Areas Commun., vol. 21, no. 5, pp. 829-838, June 2003.

[42] M. Kiessling, J. Speidel, I. Viering, and M. Reinhardt, "A closed-form bound on correlated MIMO channel capacity," inProc. IEEE Vehicular Technology Conference (VTC 2002-Fall), vol. 2, pp. 859-863, 24-28 Sept. 2002.

[43] S. Loyka and A. Kouki, "On the use of Jensen's inequality for MIMO channel capacity estimation," Canadian Conference on Electrical and Computer Engineering, 2001, vol. 1, pp. 475-480, 13-16 May 2001.

[44] S. Loyka and A. Kouki, "New compound upper bound on MIMO channel capacity," IEEE Commun. Lett., vol. 6, Issue: 3, pp. 96-98, Mar. 2002

[45] S. Loyka and A. Kouki, "The impact of correlation on multi-antenna system performance: correlation matrix approach," in Proc. IEEE Vehicular Technology Conference (VTC 2001 Fall), vol. 2, pp. 533-537, 7-11 Oct. 2001.

[46] M.A. Khalighi, J.-M. Brossier, G. Jourdain and K. Raoof, "On capacity of Rician MIMO channels," 12th IEEE International Symposium on Personal, Indoor and Mobile Radio Communications (PIMRIC 2001), vol. 1 , pp. A150-A154, 30 Sept.-3 Oct. 2001.

[47] A.M. Tulino, A. Lozano, S. Verdu, "Capacity of multi-antenna channels in the low-power regime," in Proc. 2002 IEEE Information Theory Workshop, pp. 192-195, 20-25 Oct. 2002.

[48] E. Jorswieck, G. Wunder, V. Jungnickel, and T. Haustein, "Inverse eigenvalue statistics for Rayleigh and Rician MIMO channels," IEE Seminar on MIMO: Communications Systems from Concept to Implementations, Ref. No. 2001/175, pp. 3/1-3/6, 12 Dec. 2001

[49] J. Ayadi, A. A. Hutter, and J. Farserotu, "On the multiple input multiple output capacity of Rician channels," The 5th International Symposium on Wireless Personal Multimedia Communications, vol. 2, pp. 402-406, 27-30 Oct. 2002.
[50] R. J. Muirhead, Aspects of Multivariate Statistical Theory. New York, John Wiley \& Sons Inc., 1982

[51] R. A. Horn and C.R. Johnson, Matrix Theory. Cambridge, Cambridge University Press, 1985.

[52] A. Edelman, Eigenvalues and Condition Numbers of Random Matrices, PhD thesis, MIT, 1989.

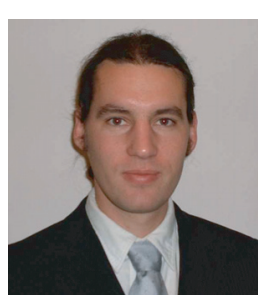

Guillaume Lebrun (S'01-M'04) received his diplôme d'ingénieur from the Ecole Nationale Supérieure des Télécommunications (ENST), Paris, France, in 2001. From 2001 to 2003 he was a $\mathrm{Ph} . \mathrm{D}$. student at Victoria University, Melbourne, Australia. He is currently with France Telecom and his research interests include OFDM, MIMO, information theory, signal processing, WiFi and WiMAX systems.

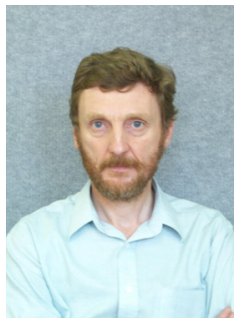

Michael Faulkner (M'84) received the B.Sc.(Eng) from Queen Mary College, London University, UK, in 1970, the M.E. degree from the University of New South Wales, Australia in 1978, and the $\mathrm{PhD}$ from University of Technology Sydney in 1993.

From 1972 to 1975 he was with STC (now Alcatel) Australia. From 1975 to 1977 he was with the University of New South Wales, and since then as a lecturer and now professor at Victoria University, Melbourne, Australia where he is director of the centre for Telecommunications and Micro-Electronics. Between 1988 and 2005 he spent five periods at Lund University, Sweden. He was co-recipient of the IEE's 1997 IERE prize for a paper on amplifier linearisation. His current interests are, signal processing, radio technology, radio systems and MIMO/OFDM.

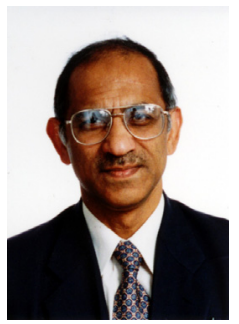

Mansoor Shafi received the B.Sc (Engineering) in Electrical Engineering from Engineering University, Lahore, Pakistan and the $\mathrm{PhD}$ degree from the University of Auckland, Auckland New Zealand in 1970 and 1979 respectively. From 1975-1979 he was a Junior Lecturer at the University of Auckland. Since 1979 he has been with Telecom New Zealand, where he now holds the position of Principal Advisor Wireless Systems. His research interests are in Wireless Communications. He has published widely in IEEE Journals and IEEE Conferences in the areas of Radio Propagation, Signal Processing, MIMO Systems, Adaptive Equalization etc. He was a guest editor of the IEEE JSAC special issue on MIMO systems published in April 2003. His co-authored, paper, "From Theory to Practice: An Overview of MIMO Space-Time Coded Wireless Systems", published in JSAC April 2003 has won the IEEE Communications Society best tutorial paper award in 2004. Mansoor is a Fellow of the IEEE and is an Adjunct Professor at Canterbury University. He was a Co-chair of the ICC 2005 Wireless Communications Symposium, held in Seoul. In Telecom New Zealand his role is to advise the Management on the future directions of Wireless Technologies and Standards.

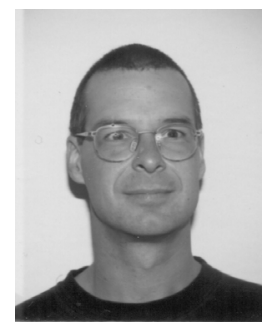

Peter J. Smith received the B.Sc degree in Mathematics and the Ph.D degree in Statistics from the University of London, London, U.K., in 1983 and 1988, respectively. From 1983 to 1986 he was with the Telecommunications Laboratories at GEC Hirst Research Centre. From 1988 to 2001, he was a Lecturer in Statistics and Consulting Statistician at Victoria University of Wellington, Wellington, New Zealand. He is currently an Associate Professor in the Department of Electrical and Computer Engineering at the University of Canterbury, Christchurch, New Zealand. His research interests include the statistical aspects of design and analysis for communication systems, especially antenna arrays, mobile radio and MIMO systems. 